\title{
EXISTENCE OF FAST POSITIVE SEMI-WAVEFRONT SOLUTIONS TO MONOSTABLE INTEGRO-DIFFERENTIAL EQUATIONS WITH DELAY
}

\author{
MAITERE AGUERREA AND ROBERT HAKL
}

Received 21 December, 2018

\begin{abstract}
We establish the existence of fast positive semi-wavefront solutions to a delay integrodifferential problem

$$
c u^{\prime}(t)=J \star u(t)-u(t)+f(u(t-h)), \quad t \in \mathbb{R}, \quad u(-\infty)=0,
$$

where the asymmetric kernel $J$ is exponentially bounded, the nonlinearity $f \in C^{1}([0,+\infty) ; \mathbb{R})$ is monostable, $h \geq 0$, and $c>0$.

2010 Mathematics Subject Classification: 34K05; 34K10; 34K12; 34K25
\end{abstract}

Keywords: Functional differential equation, positive semi-wavefront, monostable integro-differential equation

\section{INTRODUCTION}

The main object of study in this paper is a time-delayed integro-differential equation

$$
c u^{\prime}(t)=J \star u(t)-u(t)+f(u(t-h)) \quad \text { for } t \in \mathbb{R},
$$

where $h \geq 0, c>0$, and the non-negative averaging kernel $J$ satisfies

$$
J \star u(t)=\int_{\mathbb{R}} J(x) u(t-x) d x, \quad \int_{\mathbb{R}} J(x) d x=1, \quad \int_{\mathbb{R}} J(x)|x| d x<+\infty,
$$

and there exists $\lambda>0$ such that

$$
\int_{-\infty}^{0} J(x) e^{-\lambda x} d x<+\infty
$$

Further, we suppose that $f \in C^{1}([0,+\infty)$; $\mathbb{R}), f(0)=0, f(1)=0, f(x)>0$ for $x \in(0,1), f(x)<0$ for $x>1$. By a solution to (1.1) we understand a continuously differentiable function defined on the whole real axis and satisfying (1.1) at every point $t \in \mathbb{R}$.

Supported by RVO: 67985840 . 
Integro-differential models as (1.1) and its versions without delay are using when long distance dispersal events are considered. In ecology, long distance dispersal events are suspected to deeply modify the dynamics of a population [5]. Many works have shown, the phenomena observed in biological and ecological models depend not only on the present state but also on some past occurrences. The delay effect induces an important change in some predictions. For example, in population dynamics, the time delays effect the prediction of population expansions speed [13]. On the other hand, several studies also indicate that asymmetric kernels might appear in the population modeling in a natural way [16]. So, we are interested in understanding better the effect of delay in models as (1.1) when the kernel $J$ is asymmetric.

If we take $h=0$ in (1.1), we obtain an integro-differential equation without delay. Such kinds of equations appears in various biological and ecological models (e.g. population dynamics). The existence, uniqueness and propagation properties of traveling wave solutions for equation (1.1) have been investigated in a series of papers where different geometric and smoothness conditions on $J$ and $f$ were assumed (see, e.g. $[1-4,6-8,18])$. The problem of existence/nonexistence and existence of the minimal speed of wavefronts $(u(-\infty)=0$ and $u(+\infty)=\kappa)$ were considered in [7,8,18], and the propagation properties and the effect of the dispersal heavy tails in $[2-4,9]$, by means of different methods. In the mentioned papers, the results require several conditions on kernels (compactly supported, exponential tails or algebraic tails, symmetrical/asymmetric) and nonlinearities $\left(f^{\prime}(0)>0,0<f(s) \leqslant f^{\prime}(0) s, f^{\prime}(1)<0\right)$. In such cases the propagation can occur with the constant (kernel is exponentially bounded) or accelerated (kernel with heavy tails) speed.

As far as we know, if $h>0$, very few theoretical studies are devoted to integrodifferential equations with delay $[12,14,17,19]$. All these works have studied traveling wavefront solutions to equation

$$
c u^{\prime}(t)=(J \star u(t)-u(t))-d u+b(u(t-h)) \quad \text { for } t \in \mathbb{R},
$$

where $d$ is the death rate, $b$ is the birth function. In these research, using different methods (weighted energy method, the comparison principles, by constructing proper upper and lower solutions), the existence/nonexistence, stability and uniqueness of traveling wavefront solutions to (1.2) were obtained, assuming the typical FisherKPP condition on nonlinear function $b\left(0<b(s) \leq b^{\prime}(0) s\right)$, considering monotonicity or quasi-monotonicity conditions and symmetric kernels. Note that, if $h>0$, model (1.1) cannot be deduced from equation (1.2).

In this paper, we present an analytic results on the existence of waves in delayed equations which include model (1.1). We will use the methods based on the general theory of boundary value problems for functional differential equations to get the existence of fast semi-wavefront solutions to (1.1) with asymmetric kernel, where the typical conditions on $f^{\prime}(0)$ and $f^{\prime}(1)$ are not required.

Below, we present our main results. 
Theorem 1. For every $c>0$ sufficiently large there exists a positive solution $u$ to the equation (1.1) satisfying

$$
\lim _{t \rightarrow-\infty} u(t)=0, \quad e^{-\lambda h} \leq \liminf _{t \rightarrow+\infty} u(t) \leq \sup _{t \in \mathbb{R}} u(t) \leq 1+f_{0}\left(e^{\frac{h}{c}}-1\right),
$$

where $f_{0}=\max \{f(s): s \in[0,1]\}$.

Corollary 1. For every $c>0$ sufficiently large there exists a positive solution $u$ to the equation (1.1) with $h=0$ satisfying

$$
\lim _{t \rightarrow-\infty} u(t)=0, \quad \lim _{t \rightarrow+\infty} u(t)=1 .
$$

Remark 1 . We observe that Corollary 1 shows that the special conditions $f^{\prime}(0)>0$ and $f^{\prime}(1)<0$ (steady state 1 is stable) are not necessary to prove the existence of fast wavefront solutions to (1.1) when $h=0$.

The paper is organized as follows. In Section 2, some auxiliary propositions are given and the existence of fast positive semi-wavefronts is proven. In the last section, Section 3, the existence theorem is applied to some models.

\section{AUXILIARY PROPOSITIONS}

In what follows we will use the following notation. $C([0,+\infty) ; \mathbb{R}), C(\mathbb{R} ; \mathbb{R})$, $C([-N, N] ; \mathbb{R})$, resp. $C^{1}([0,+\infty) ; \mathbb{R})$ are standard spaces of continuous, resp. continuously differentiable functions. If $u \in C([-N, N] ; \mathbb{R})$, then $\|u\|_{C}=\max \{|u(t)|$ : $t \in[-N, N]\}$. Put

$$
\begin{gathered}
f_{0} \stackrel{\text { def }}{=} \max \{f(s): s \in[0,1]\}, \quad x_{c} \stackrel{\text { def }}{=} 1+f_{0}\left(e^{\frac{h}{c}}-1\right), \\
f_{c} \stackrel{\text { def }}{=} \max \left\{\left|f^{\prime}(s)\right|: s \in\left[0, x_{c}\right]\right\} .
\end{gathered}
$$

Obviously, according to the assumptions laid on $f$, we have that $f(x)+f_{c} x \geq 0$ for $x \in\left[0, x_{c}\right]$.

Further we define auxiliar functions $\bar{f} \in C([0,+\infty) ; \mathbb{R})$ and $\widetilde{f} \in C(\mathbb{R} ; \mathbb{R})$ by

$$
\begin{gathered}
\bar{f}(x)= \begin{cases}f(x) & \text { for } x \in\left[0, x_{c}\right], \\
f\left(x_{c}\right)-f_{c}\left(x-x_{c}\right) & \text { for } x>x_{c},\end{cases} \\
\widetilde{f}(x)= \begin{cases}0 & \text { for } x<0, \\
\bar{f}(x)+f_{c} x & \text { for } x \geq 0,\end{cases}
\end{gathered}
$$

Note that $\widetilde{f}$ is a bounded non-negative function.

For every $N>0$ we define an operator $\theta_{N}: C([-N, N] ; \mathbb{R}) \rightarrow C(\mathbb{R} ; \mathbb{R})$ by

$$
\theta_{N}(u)(t)= \begin{cases}u(-N) & \text { for } t<-N, \\ u(t) & \text { for } t \in[-N, N], \\ u(N) & \text { for } t>N,\end{cases}
$$


and let $\ell_{N}^{+}, \ell_{N}^{-}: C([-N, N] ; \mathbb{R}) \times \mathbb{R}_{+} \rightarrow C([-N, N] ; \mathbb{R})$ be operators given by

$$
\begin{aligned}
& \ell_{N}^{+}(u, \mu)(t)=\int_{\mathbb{R}} J(x) \theta_{N}(u)(t-x) d x+\mu \theta_{N}(u)(t-h), \\
& \ell_{N}^{-}(u, \mu)(t)=u(t)+\mu \theta_{N}(u)(t-h) .
\end{aligned}
$$

It can be easily verified that the operators $\ell_{N}^{+}$and $\ell_{N}^{-}$are linear positive operators in the first variable, i.e., for every fixed $\mu_{0} \in \mathbb{R}_{+}$, the operators $\ell_{N}^{+}\left(\cdot, \mu_{0}\right), \ell_{N}^{-}\left(\cdot, \mu_{0}\right)$ : $C([-N, N] ; \mathbb{R}) \rightarrow C([-N, N] ; \mathbb{R})$ are linear and transforms the set $C\left([-N, N] ; \mathbb{R}_{+}\right)$ into the set $C\left([-N, N] ; \mathbb{R}_{+}\right)$. Moreover, the operator $\ell_{N}^{-}\left(\cdot, \mu_{0}\right)$ is a Volterra operator (with respect to the point $-N)$, i.e., for every $t_{0} \in(-N, N]$ the equality

$$
u(t)=0 \quad \text { for } t \in\left[-N, t_{0}\right]
$$

implies

$$
\ell_{N}^{-}\left(u, \mu_{0}\right)(t)=0 \quad \text { for } t \in\left[-N, t_{0}\right] .
$$

Lemma 1. Let $\mu_{0} \geq 0$ and let $c>0$ satisfy

$$
c \lambda \geq \int_{0}^{+\infty} J(x) d x+\int_{-\infty}^{0} J(x) e^{-\lambda x} d x+\mu_{0} .
$$

Then, for every $N>h$, the function $\gamma(t)=e^{\lambda t}$ for $t \in[-N, N]$ satisfies

$$
c \gamma^{\prime}(t) \geq \ell_{N}^{+}(\gamma, \mu)(t) \quad \text { for } t \in[-N, N], \quad \mu \in\left[0, \mu_{0}\right] .
$$

Proof. According to the definition of $\ell_{N}^{+}$we have that

$$
\begin{aligned}
\ell_{N}^{+}(\gamma, \mu)(t)=e^{\lambda t}\left(e^{-\lambda(t+N)}\right. & \int_{t+N}^{+\infty} J(x) d x+e^{-\lambda(t-N)} \int_{-\infty}^{t-N} J(x) d x \\
& \left.+\int_{t-N}^{t+N} J(x) e^{-\lambda x} d x+p(t)\right) \quad \text { for } t \in[-N, N],
\end{aligned}
$$

where

$$
p(t)= \begin{cases}\mu e^{-\lambda(t+N)} & \text { for } t \in[-N,-N+h), \\ \mu e^{-\lambda h} & \text { for } t \in[-N+h, N] .\end{cases}
$$

It can be easily verified that the function in the parenthesis is non-increasing with respect to $t$. Therefore, we get

$$
\begin{aligned}
& \ell_{N}^{+}(\gamma, \mu)(t) \leq e^{\lambda t}\left(\int_{0}^{+\infty} J(x) d x+e^{2 \lambda N} \int_{-\infty}^{-2 N} J(x) d x+\int_{-2 N}^{0} J(x) e^{-\lambda x} d x+\mu\right) \\
& \leq e^{\lambda t}\left(\int_{0}^{+\infty} J(x) d x+\int_{-\infty}^{0} J(x) e^{-\lambda x} d x+\mu_{0}\right) \text { for } t \in[-N, N], \mu \in\left[0, \mu_{0}\right] .
\end{aligned}
$$

Now, using (2.1) in the last inequality we arrive at (2.2). 
By a direct calculation one can verify the following assertion.

Lemma 2. Let $\mu_{0} \geq 0$ and let $c>0$ satisfy

$$
c \lambda \geq 1+\mu_{0} e^{\lambda h}
$$

Then, for every $N>h$, the function $\beta(t)=e^{-\lambda t}$ for $t \in[-N, N]$ satisfies

$$
c \beta^{\prime}(t) \leq-\ell_{N}^{-}(\beta, \mu)(t), \quad \text { for } t \in[-N, N], \quad \mu \in\left[0, \mu_{0}\right] .
$$

Remark 2. Note that $x_{c}$ and $f_{c}$ are nonincreasing with respect to $c$. Therefore, there exists $c_{*}>0$ such that, for every $c \geq c_{*}$, we have that

$$
\begin{gathered}
c \lambda \geq \int_{0}^{+\infty} J(x) d x+\int_{-\infty}^{0} J(x) e^{-\lambda x} d x+f_{c}, \\
c \lambda \geq 1+f_{c} e^{\lambda h} .
\end{gathered}
$$

Thus, in what follows, by $c_{*}$ we refer to the number described above.

Now we introduce some a priori estimates.

Lemma 3. Let $N>h, c \geq c_{*}$, and let $u$ be a non-negative solution to the equation

$$
c u^{\prime}(t)=\ell_{N}^{+}(u, 0)(t)-u(t)+\bar{f}\left(\theta_{N}(u)(t-h)\right) \quad \text { for } t \in[-N, N]
$$

such that $u(-N) \leq e^{-\lambda h}$. Then

$$
u(-N)=\min \{u(t): t \in[-N, N]\} .
$$

Proof. Suppose on the contrary that $u(-N)>\min \{u(t): t \in[-N, N]\}$. Then there exists $t_{0} \in(-N, N]$ such that

$$
u\left(t_{0}\right)=\min \{u(t): t \in[-N, N]\}<e^{-\lambda h}, \quad u(t)>u\left(t_{0}\right) \quad \text { for } t \in\left[-N, t_{0}\right) .
$$

Obviously,

$$
\begin{aligned}
c u^{\prime}(t)=\ell_{N}^{+}(u, 0)(t)-\ell_{N}^{-}\left(u, f_{c}\right)(t)+\widetilde{f}\left(\theta_{N}(u)(t-h)\right) & \\
& \geq-\ell_{N}^{-}\left(u, f_{c}\right)(t) \quad \text { for } t \in\left[-N, t_{0}\right] .
\end{aligned}
$$

According to Lemma 2, the function $\beta(t)=e^{-\lambda t}$ satisfies

$$
c \beta^{\prime}(t) \leq-\ell_{N}^{-}\left(\beta, f_{c}\right)(t) \quad \text { for } t \in\left[-N, t_{0}\right] .
$$

Consequently (see [10, Theorem 1.5]), we find that

$$
u(t) \leq \frac{u\left(t_{0}\right)}{\beta\left(t_{0}\right)} \beta(t)=u\left(t_{0}\right) e^{-\lambda\left(t-t_{0}\right)} \quad \text { for } t \in\left[-N, t_{0}\right] .
$$

In particular,

$$
\theta_{N}(u)(t) \leq u\left(t_{0}\right) e^{\lambda(h+\varepsilon)} \leq 1 \quad \text { for } t \in\left[t_{0}-(h+\varepsilon), t_{0}\right],
$$

for a suitable positive $\varepsilon \leq t_{0}+N$, which implies

$$
\bar{f}\left(\theta_{N}(u)(t-h)\right) \geq 0 \quad \text { for } t \in\left[t_{0}-\varepsilon, t_{0}\right] .
$$


Set $w(t)=u(t)-u\left(t_{0}\right)$ for $t \in[-N, N]$. Then, obviously, $w(t) \geq 0$ and

$$
c w^{\prime}(t)=\ell_{N}^{+}(w, 0)(t)-w(t)+\bar{f}\left(\theta_{N}(u)(t-h)\right) \geq-w(t) \quad \text { for } t \in\left[t_{0}-\varepsilon, t_{0}\right] .
$$

Consequently, $u(t)=u\left(t_{0}\right)$ for $t \in\left[t_{0}-\varepsilon, t_{0}\right]$, a contradiction.

Lemma 4. Let $N>h, c \geq c_{*}$, and let $u$ be a non-negative solution to (2.6) such that $u(-N) \leq e^{-\lambda h}$. Then

$$
\|u\|_{C} \leq x_{c} .
$$

Proof. According to Lemma 3, there exists $t_{0} \in(-N, N]$ such that $u\left(t_{0}\right)=\|u\|_{C}$ and

$$
0 \leq c u^{\prime}\left(t_{0}\right)=\int_{\mathbb{R}} J(x)\left[\theta_{N}(u)\left(t_{0}-x\right)-u\left(t_{0}\right)\right] d x+\bar{f}\left(\theta_{N}(u)\left(t_{0}-h\right)\right) .
$$

Therefore, $\bar{f}\left(\theta_{N}(u)\left(t_{0}-h\right)\right) \geq 0$, i.e. $\theta_{N}(u)\left(t_{0}-h\right) \leq 1$ and assuming $\|u\|_{C}>x_{c}$, there exists $t_{1} \in\left[t_{0}-h, t_{0}\right)$ such that $u\left(t_{1}\right)=1$. Obviously, $t_{1} \geq-N$. Then (2.6) implies

$$
c u^{\prime}(t) \leq u\left(t_{0}\right)-u(t)+f_{0} \quad \text { for } t \in\left[t_{1}, t_{0}\right]
$$

whence we obtain

$$
u\left(t_{0}\right) e^{\frac{t_{0}}{c}} \leq u\left(t_{1}\right) e^{\frac{t_{1}}{c}}+\left[u\left(t_{0}\right)+f_{0}\right]\left(e^{\frac{t_{0}}{c}}-e^{\frac{t_{1}}{c}}\right)
$$

and, consequently,

$$
u\left(t_{0}\right) \leq 1+f_{0}\left(e^{\frac{h}{c}}-1\right)=x_{c},
$$

a contradiction.

Lemma 5. Let $N>h, c \geq c_{*}$ and let $u$ be a non-negative solution to the equation

$$
c u^{\prime}(t)=\ell_{N}^{+}(u, 0)(t)-u(t)+f\left(\theta_{N}(u)(t-h)\right) \quad \text { for } t \in[-N, N]
$$

such that $\|u\|_{C} \leq x_{c}$. Then

$$
u(0) \leq u(-N) e^{\lambda N} .
$$

Proof. According to (2.7) and the assumption $f \in C^{1}([0,+\infty)$; $\mathbb{R})$ we have that

$$
c u^{\prime}(t) \leq \ell_{N}^{+}\left(u, f_{c}\right)(t) \quad \text { for } t \in[-N, N] .
$$

Further, according to (2.4) and Lemma 1, the function $\gamma(t)=e^{\lambda t}$ satisfies

$$
c \gamma^{\prime}(t) \geq \ell_{N}^{+}\left(\gamma, f_{c}\right)(t) \quad \text { for } t \in[-N, N] .
$$

Consequently (see [10, Theorem 1.1]), we find that

$$
u(t) \leq \frac{u(-N)}{\gamma(-N)} \gamma(t)=u(-N) e^{\lambda(t+N)} \quad \text { for } t \in[-N, N] .
$$

In particular, for $t=0$ we obtain the assertion of the lemma. 
Lemma 6. Let $N>h, c \geq c_{*}$, and let $u_{1}$ and $u_{2}$ be non-negative solutions to the equation (2.7) such that

$$
\left\|u_{i}\right\|_{C} \leq x_{c} \quad(i=1,2), \quad u_{1}(-N) \leq u_{2}(-N) .
$$

Then

$$
u_{1}(t) \leq u_{2}(t) \quad \text { for } t \in[-N, N] .
$$

Proof. Set $w(t)=u_{1}(t)-u_{2}(t)$ for $t \in[-N, N]$. Then we have

$$
\begin{aligned}
& c w^{\prime}(t)=\ell_{N}^{+}(w, 0)(t)-w(t)+f\left(\theta_{N}\left(u_{1}\right)(t-h)\right)-f\left(\theta_{N}\left(u_{2}\right)(t-h)\right) \\
& \quad=\ell_{N}^{+}(w, 0)(t)-w(t)+p(t) \theta_{N}(w)(t-h) \quad \text { for } t \in[-N, N], \quad w(-N) \leq 0,
\end{aligned}
$$

where

$$
p(t)= \begin{cases}\frac{f\left(\theta_{N}\left(u_{1}\right)(t-h)\right)-f\left(\theta_{N}\left(u_{2}\right)(t-h)\right)}{\theta_{N}\left(u_{1}\right)(t-h)-\theta_{N}\left(u_{2}\right)(t-h)} & \text { if } \theta_{N}\left(u_{1}\right)(t-h) \neq \theta_{N}\left(u_{2}\right)(t-h), \\ 0 & \text { otherwise. }\end{cases}
$$

Obviously, $|p(t)| \leq f_{c}$ for $t \in[-N, N]$ and according to (2.4), (2.5), and Lemmas 1 and 2, the functions $\gamma(t)=e^{\lambda t}$ and $\beta(t)=e^{-\lambda t}$ satisfy

$$
\begin{gathered}
c \gamma^{\prime}(t) \geq \ell_{N}^{+}\left(\gamma, f_{c}\right)(t) \geq \ell_{N}^{+}(\gamma, 0)(t)+[p(t)]_{+} \theta_{N}(\gamma)(t-h) \quad \text { for } t \in[-N, N], \\
c \beta^{\prime}(t) \leq-\ell_{N}^{-}\left(\beta, f_{c}\right)(t) \leq-\beta(t)-[p(t)]_{-} \theta_{N}(\beta)(t-h) \quad \text { for } t \in[-N, N] .
\end{gathered}
$$

However, according to $[10$, Theorems $1.1,1.2,1.4]$, the latter inequalities imply that $w(t) \leq 0$ for $t \in[-N, N]$, i.e. $u_{1}(t) \leq u_{2}(t)$ for $t \in[-N, N]$.

Remark 3. Note that according to Lemma 6, the problem

$$
c u^{\prime}(t)=\ell_{N}^{+}(u, 0)(t)-u(t)+f\left(\theta_{N}(u)(t-h)\right), \quad u(-N)=r
$$

with $r \in\left[0, x_{c}\right]$ has at most one solution such that $\|u\|_{C} \leq x_{c}$ provided $N>h$ and $c \geq c_{*}$.

Lemma 7. Let $c \geq c_{*}$ and $u_{0} \in\left(0, e^{-\lambda h}\right]$. Then, for every $N>h$, there exists a positive solution $u \in C([-N, N] ; \mathbb{R})$ to the problem

$$
c u^{\prime}(t)=\ell_{N}^{+}(u, 0)(t)-u(t)+f\left(\theta_{N}(u)(t-h)\right), \quad u(0)=u_{0}
$$

such that $\|u\|_{C} \leq x_{c}$.

Proof. Let $N>h$ be arbitrary but fixed. According to (2.4), (2.5), and Lemmas 1 and 2, the functions $\gamma(t)=e^{\lambda t}$ and $\beta(t)=e^{-\lambda t}$ satisfy

$$
c \gamma^{\prime}(t) \geq \ell_{N}^{+}(\gamma, 0)(t), \quad c \beta^{\prime}(t) \leq-\ell_{N}^{-}\left(\beta, f_{c}\right)(t) \quad \text { for } t \in[-N, N] .
$$

Consequently (see [10, Theorems 1.1, 1.2, 1.4]), the homogeneous problem

$$
c u^{\prime}(t)=\ell_{N}^{+}(u, 0)(t)-\ell_{N}^{-}\left(u, f_{c}\right)(t), \quad u(-N)=0
$$

has only the trivial solution and thus, the problem

$$
c u^{\prime}(t)=\ell_{N}^{+}(u, 0)(t)-\ell_{N}^{-}\left(u, f_{c}\right)(t)+\widetilde{f}\left(\theta_{N}(u)(t-h)\right), \quad u(-N)=r
$$


has a solution $u_{r}$ for every $r \geq 0$ (see [11, Theorem 3.1]). Moreover, since $\widetilde{f}$ is a non-negative function, the solution $u_{r}$ is also non-negative on $[-N, N]$ (see $[10$, Theorem 1.4]). Consequently, $u_{r}$ is a solution to

$$
c u^{\prime}(t)=\ell_{N}^{+}(u, 0)(t)-u(t)+\bar{f}\left(\theta_{N}(u)(t-h)\right), \quad u(-N)=r .
$$

Now we will show that there exists $r_{0} \in\left[u_{0} e^{-\lambda N}, u_{0}\right]$ such that the corresponding solution $u_{r_{0}}$ admits $u_{r_{0}}(0)=u_{0}$.

According to Lemmas 3 and 4 , for $r \leq e^{-\lambda h}$ we have that

$$
u_{r}(-N) \leq u_{r}(t) \leq x_{c} \quad \text { for } t \in[-N, N]
$$

and so, for $r_{1}=u_{0}$ we get

$$
u_{0}=u_{r_{1}}(-N) \leq u_{r_{1}}(0) .
$$

Moreover, all the solutions $u_{r}$ with $0<r \leq e^{-\lambda h}$ are, obviously, also positive solutions to the equation (2.7). Consequently, according to Lemma 5, for $r_{2}=u_{0} e^{-\lambda N}$ we have that

$$
u_{r_{2}}(0) \leq u_{r_{2}}(-N) e^{\lambda N}=u_{0} .
$$

Furthermore, Remark 3 yields the uniqueness of every solution $u_{r}\left(r \leq e^{-\lambda h}\right)$ and thus, the continuous dependence on parameter (see [15, Theorem 2.1]) implies the existence of $r_{0} \in\left[u_{0} e^{-\lambda N}, u_{0}\right]$ such that $u_{r_{0}}(0)=u_{0}$.

Lemma 8. Let $c \geq c_{*}$ and let $u$ be a non-negative solution to (1.1) such that $u(t) \leq x_{c}$ for $t \in \mathbb{R}$. Then

$$
u(s) \leq u(t) e^{\lambda(t-s)} \quad \text { for } s, t \in \mathbb{R}, s \leq t .
$$

Proof. It follows from (2.5) that $\beta(t)=e^{-\lambda t}$ satisfies

$$
c \beta^{\prime}(t) \leq-\beta(t)-f_{c} \beta(t-h) \quad \text { for } t \in \mathbb{R} .
$$

Further, from (1.1) it follows that

$$
\begin{aligned}
c u^{\prime}(t)=J \star u(t)-u(t)-f_{c} u(t-h)+\widetilde{f}(u(t-h)) & \\
& \geq-u(t)-f_{c} u(t-h) \quad \text { for } t \in \mathbb{R} .
\end{aligned}
$$

Now, let $t_{0} \in \mathbb{R}$ be arbitrary but fixed. Obviously,

$$
u(t)=o(\beta(t)) \quad \text { as } \quad t \rightarrow-\infty .
$$

Therefore, there exists $t_{1} \leq t_{0}$ such that

$$
\mu \stackrel{\text { def }}{=} \sup \left\{\frac{u(t)}{\beta(t)}: t \leq t_{0}\right\}=\frac{u\left(t_{1}\right)}{\beta\left(t_{1}\right)},
$$

and so

$$
\mu \beta(t)-u(t) \geq 0 \quad \text { for } t \leq t_{0}, \quad \mu \beta\left(t_{1}\right)-u\left(t_{1}\right)=0,
$$

and

$$
c\left(\mu \beta^{\prime}(t)-u^{\prime}(t)\right) \leq-(\mu \beta(t)-u(t))-f_{c}(\mu \beta(t-h)-u(t-h)) \leq 0 \quad \text { for } t \leq t_{0} .
$$


Therefore, $\mu \beta\left(t_{0}\right)=u\left(t_{0}\right)$ and so

$$
u(t) \leq \frac{u\left(t_{0}\right)}{\beta\left(t_{0}\right)} \beta(t)=u\left(t_{0}\right) e^{\lambda\left(t_{0}-t\right)} \quad \text { for } t \leq t_{0} .
$$

Since $t_{0}$ was chosen arbitrarily, we get the assertion of the lemma.

Lemma 9. Let $c \geq c_{*}$. Then, for every $u_{0} \in\left(0, e^{-\lambda h}\right]$, there exists a positive solution $u$ to the equation (1.1) such that

$$
u(0)=u_{0}, \quad \sup _{t \in \mathbb{R}} u(t) \leq x_{c} .
$$

Proof. According to Lemma 7 there exist an increasing sequence of real numbers $N_{i}(i \in \mathbb{N})$ such that

$$
N_{1}>h, \quad \lim _{i \rightarrow+\infty} N_{i}=+\infty
$$

and a sequence of positive solutions $u_{N_{i}}:\left[-N_{i}, N_{i}\right] \rightarrow \mathbb{R}$ to (2.8) (with $N=N_{i}$ ) satisfying $\left\|u_{N_{i}}\right\|_{C} \leq x_{c}(i \in \mathbb{N})$. Therefore, $\left\{\theta_{N_{i}}\left(u_{N_{i}}\right)\right\}_{i=1}^{+\infty}$ is a sequence of positive continuous functions defined on $\mathbb{R}$, uniformly bounded by $x_{c}$, and equicontinuous on every compact interval contained in $\mathbb{R}$. Without loss of generality we can assume that there exists a continuous function $u$ such that

$$
\lim _{i \rightarrow+\infty} \theta_{N_{i}}\left(u_{N_{i}}\right)(t)=u(t) \quad \text { uniformly on every compact subinterval of } \mathbb{R} \text {. }
$$

As a limit of $\theta_{N_{i}}\left(u_{N_{i}}\right)$, the function $u$ satisfies $0 \leq u(t) \leq x_{c}$ for $t \in \mathbb{R}$, which implies that

$$
\lim _{i \rightarrow+\infty} \int_{I}\left|\ell_{N_{i}}^{+}\left(u_{N_{i}}, 0\right)(t)-J \star u(t)\right| d t=0
$$

for every compact interval $I \subset \mathbb{R}$. Consequently, $u$ is a non-negative nontrivial $\left(u(0)=u_{0}>0\right)$ solution to (1.1).

To show that $u$ is a positive function, assume on the contrary that $u$ vanishes at some point $t_{0} \in \mathbb{R}$. According to Lemma 8 we have $u(t)=0$ for $t \leq t_{0}$. Moreover,

$$
c u^{\prime}(t)=J \star u(t)-u(t)+f(u(t-h)) \leq J \star u(t)+f_{c} u(t-h) \text { for } t \in \mathbb{R} .
$$

On the other hand, according to (2.4), the function $\gamma(t)=e^{\lambda t}$ satisfies

$$
c \gamma^{\prime}(t) \geq J \star \gamma(t)+f_{c} \gamma(t-h) \quad \text { for } t \in \mathbb{R}
$$

and

$$
u(t)=o(\gamma(t)) \quad \text { as } \quad t \rightarrow+\infty .
$$

Consequently, there exists $t_{1} \geq t_{0}$ such that

$$
\mu=\sup \left\{\frac{u(t)}{\gamma(t)}: t \geq t_{0}\right\}=\frac{u\left(t_{1}\right)}{\gamma\left(t_{1}\right)}
$$

and so

$$
\mu \gamma(t)-u(t) \geq 0 \quad \text { for } t \geq t_{0}, \quad \mu \gamma\left(t_{1}\right)-u\left(t_{1}\right)=0 .
$$


Since $u(t)=0$ for $t \leq t_{0}$, we have $\mu \gamma(t)-u(t) \geq 0$ for $t \in \mathbb{R}$ and

$c\left(\mu \gamma^{\prime}(t)-u^{\prime}(t)\right) \geq J \star(\mu \gamma(t)-u(t))+f_{c}(\mu \gamma(t-h)-u(t-h)) \geq 0$ for $t \geq t_{0}$.

Therefore, $\mu \gamma\left(t_{0}\right)=u\left(t_{0}\right)=0$ and so $\mu=0$. Thus, $u \equiv 0$ on $\mathbb{R}$ that contradicts $u(0)=u_{0}>0$.

Lemma 10. Let $c \geq c_{*}$ and let $u$ be a positive solution to (1.1) such that

$$
\inf _{t \in \mathbb{R}} u(t)<e^{-\lambda h}, \quad \sup _{t \in \mathbb{R}} u(t) \leq x_{c} .
$$

Then

$$
u(t)>\inf _{s \in \mathbb{R}} u(s) \quad \text { for } t \in \mathbb{R} .
$$

Proof. Assume on the contrary that there exists $t_{0} \in \mathbb{R}$ such that

$$
u\left(t_{0}\right)=\inf _{t \in \mathbb{R}} u(t) .
$$

Then, according to Lemma 8 we have that $u\left(t_{0}-h\right)<1$, and thus $f\left(u\left(t_{0}-h\right)\right)>0$. On the other hand,

$$
0=c u^{\prime}\left(t_{0}\right)=\int_{\mathbb{R}} J(x)\left[u\left(t_{0}-x\right)-u\left(t_{0}\right)\right] d x+f\left(u\left(t_{0}-h\right)\right) \geq f\left(u\left(t_{0}-h\right)\right),
$$

a contradiction.

Lemma 11. For any non-negative bounded function $u$ we have

$$
\liminf _{t \rightarrow \pm \infty} J \star u(t) \geq \liminf _{t \rightarrow \pm \infty} u(t) .
$$

Proof. Obviously, for every $N>0$ we have that

$$
\begin{aligned}
\int_{\mathbb{R}} J(x) u(t-x) d x \geq & \int_{-\infty}^{N} J(x) u(t-x) d x \\
& \geq \int_{-\infty}^{N} J(x) d x \inf \{u(s): s \in[t-N,+\infty)\} \quad \text { for } t \in \mathbb{R} .
\end{aligned}
$$

Consequently,

$$
\liminf _{t \rightarrow+\infty} J \star u(t) \geq \int_{-\infty}^{N} J(x) d x \liminf _{t \rightarrow+\infty} u(t) \quad \text { for } N>0 .
$$

Thus, the assertion of the lemma follows as $N \rightarrow+\infty$. The case $t \rightarrow-\infty$ can be proven analogously.

Lemma 12. Let $c \geq c_{*}$ satisfy

$$
c>\int_{\mathbb{R}} J(x)|x| d x
$$


and let $u$ be a positive solution to (1.1) such that $u(t) \leq x_{c}$ for $t \in \mathbb{R}$ and

$$
\liminf _{t \rightarrow+\infty} u(t)<e^{-\lambda h}, \quad \text { resp. } \quad \liminf _{t \rightarrow-\infty} u(t)<e^{-\lambda h} .
$$

Then

$$
\lim _{t \rightarrow+\infty} u(t)=0, \quad \text { resp. } \quad \lim _{t \rightarrow-\infty} u(t)=0 .
$$

Proof. We will proof the lemma as $t \rightarrow+\infty$. For the case when $t \rightarrow-\infty$ the proof is similar. First we prove that there exists a limit of $u(t)$ as $t \rightarrow+\infty$. Assume on the contrary that

$$
u_{*} \stackrel{\text { def }}{=} \liminf _{t \rightarrow+\infty} u(t)<\limsup _{t \rightarrow+\infty} u(t)
$$

According to Lemma 8 we have

$$
u(t-h)<1 \quad \text { whenever } \quad u(t)<e^{-\lambda h} .
$$

Therefore, there exist $s_{n}, t_{n} \in \mathbb{R}(n \in \mathbb{N})$ and $y_{0} \in\left(u_{*}, e^{-\lambda h}\right)$ such that $s_{n}<t_{n}$,

$$
\begin{gathered}
\lim _{n \rightarrow+\infty} s_{n}=\lim _{n \rightarrow+\infty} t_{n}=+\infty, \quad \lim _{n \rightarrow+\infty} u\left(t_{n}\right)=u_{*}, \quad u\left(s_{n}\right)=y_{0}, \\
u\left(t_{n}\right) \leq u(t) \leq u\left(s_{n}\right), \quad u(t-h)<1 \quad \text { for } t \in\left[s_{n}, t_{n}\right] .
\end{gathered}
$$

Then

$$
c u^{\prime}(t) \geq \int_{\mathbb{R}} J(x)[u(t-x)-u(t)] d x \quad \text { for } t \in\left[s_{n}, t_{n}\right] .
$$

Now we show that $t_{n}-s_{n} \rightarrow+\infty$ as $n \rightarrow+\infty$. Assume on the contrary that there exists $K>0$ such that $t_{n}-s_{n} \leq K$ for $n \in \mathbb{N}$. According to Lemma 11 and (2.12), for every $\varepsilon>0$ there exists $n_{0} \in \mathbb{N}$ such that

$$
c u^{\prime}(t) \geq u_{*}-\varepsilon-u(t) \quad \text { for } t \in\left[s_{n}, t_{n}\right], \quad n \geq n_{0} .
$$

Consequently, we have that

$$
u\left(t_{n}\right) e^{\frac{t_{n}}{c}} \geq u\left(s_{n}\right) e^{\frac{s_{n}}{c}}+\left(u_{*}-\varepsilon\right)\left(e^{\frac{t_{n}}{c}}-e^{\frac{s_{n}}{c}}\right),
$$

whence we obtain

$$
u\left(t_{n}\right)-u_{*} \geq\left(y_{0}-u_{*}\right) e^{\frac{-K}{c}}-\varepsilon\left(1-e^{\frac{-K}{c}}\right) .
$$

Passing to the limit as $n \rightarrow+\infty$ in the latter equation we find that

$$
0<y_{0}-u_{*} \leq \varepsilon\left(e^{\frac{K}{c}}-1\right),
$$

whence we get a contradiction, because $\varepsilon$ can be chosen arbitrarily small. Consequently, we get that $t_{n}-s_{n} \rightarrow+\infty$ as $n \rightarrow+\infty$. Therefore, for every $N>0$ we have that $N<t_{n}-s_{n}$ provided $n$ is sufficiently large. Now

$$
c u^{\prime}(t) \geq \int_{\mathbb{R}} J(x)[u(t-x)-u(t)] d x
$$




$$
=-\int_{\mathbb{R}} J(x) x \int_{0}^{1} u^{\prime}(t-z x) d z d x \quad \text { for } t \in\left[s_{n}, t_{n}\right] .
$$

Integration of the last inequality over the interval $\left[s_{n}, t_{n}\right]$, using Fubini's theorem, yields

$$
\begin{gathered}
c\left(u\left(t_{n}\right)-y_{0}\right) \geq-\int_{\mathbb{R}} J(x) x \int_{0}^{1}\left[u\left(t_{n}-z x\right)-u\left(s_{n}-z x\right)\right] d z d x \\
\geq-\int_{0}^{N} J(x)|x| \int_{0}^{1} u\left(t_{n}-z x\right) d z d x+\int_{0}^{N} J(x)|x| d x \inf \left\{u(\xi): \xi \in\left[s_{n}-N,+\infty\right)\right\} \\
+\int_{-N}^{0} J(x)|x| d x \inf \left\{u(\xi): \xi \in\left[t_{n},+\infty\right)\right\}-\int_{-N}^{0} J(x)|x| \int_{0}^{1} u\left(s_{n}-z x\right) d z d x \\
-\left(\int_{N}^{+\infty} J(x)|x| d x+\int_{-\infty}^{-N} J(x)|x| d x\right) x_{c} \\
\geq\left(\inf \left\{u(\xi): \xi \in\left[s_{n}-N,+\infty\right)\right\}-y_{0}\right) \int_{-N}^{N} J(x)|x| d x-\varepsilon(N) \\
\geq\left(\inf \left\{u(\xi): \xi \in\left[s_{n}-N,+\infty\right)\right\}-y_{0}\right) \int_{\mathbb{R}} J(x)|x| d x-\varepsilon(N)
\end{gathered}
$$

provided $n$ is sufficiently large, where

$$
\lim _{N \rightarrow+\infty} \varepsilon(N)=0
$$

However, passing to the limit as $n \rightarrow+\infty$ in (2.13) we get

$$
\left(u_{*}-y_{0}\right)\left(c-\int_{\mathbb{R}} J(x)|x| d x\right) \geq-\varepsilon(N) \quad \text { for } N>0 .
$$

Consequently, in view of (2.14), the inequality (2.15) yields

$$
\left(u_{*}-y_{0}\right)\left(c-\int_{\mathbb{R}} J(x)|x| d x\right) \geq 0 .
$$

Thus, on account of $y_{0}>u_{*}$ we have

$$
c \leq \int_{\mathbb{R}} J(x)|x| d x
$$

which contradicts (2.11). The obtained contradiction proves that the limit of $u(t)$ as $t \rightarrow+\infty$ exists.

Now we will show that $u_{*}=\lim _{t \rightarrow+\infty} u(t)$ is equal to zero. Obviously, according to Lemma 11 we have that for every $\varepsilon>0$ there exists $t_{\varepsilon} \in \mathbb{R}$ such that

$$
c u^{\prime}(t) \geq u_{*}-\varepsilon-u(t)+f(u(t-h)) \quad \text { for } t \geq t_{\varepsilon}
$$


and the integration from $t$ to $t+1$ yields

$$
c u(t+1)-c u(t) \geq u_{*}-\varepsilon-\int_{t}^{t+1} u(s) d s+\int_{t}^{t+1} f(u(s-h)) d s \quad \text { for } t \geq t_{\varepsilon} .
$$

Passing to the limit as $t \rightarrow+\infty$ we arrive at

$$
\varepsilon \geq \lim _{t \rightarrow+\infty} \int_{t}^{t+1} f(u(s-h)) d s=f\left(u_{*}\right)
$$

since $f$ is continuous. Consequently, since $\varepsilon>0$ can be chosen arbitrarily small, $f\left(u_{*}\right)=0$ and so $u_{*}=0$.

Lemma 13. Let $c \geq c_{*}$ satisfy (2.11) and let $u$ be a positive solution to (1.1) such that $u(t) \leq x_{c}$ for $t \in \mathbb{R}$. Then

$$
\liminf _{t \rightarrow+\infty} u(t) \geq e^{-\lambda h}
$$

Proof. Assume on the contrary that

$$
\liminf _{t \rightarrow+\infty} u(t)<e^{-\lambda h} .
$$

Then according to Lemma 12 we have

$$
\lim _{t \rightarrow+\infty} u(t)=0
$$

Consequently, there exists $t_{0}>0$ such that

$$
u\left(t_{0}\right) \geq u(t), \quad u(t-h)<1 \quad \text { for } t \geq t_{0}
$$

and thus $u$ satisfies

$$
\begin{aligned}
c u^{\prime}(t) \geq \int_{\mathbb{R}} J(x)[u(t-x)-u(t)] d x & \\
& =-\int_{\mathbb{R}} J(x) x \int_{0}^{1} u^{\prime}(t-z x) d z d x \quad \text { for } t \geq t_{0} .
\end{aligned}
$$

Integrating the last inequality from $t_{0}$ to $t$ and using Fubini's theorem we obtain that

$$
\begin{aligned}
& c\left(u(t)-u\left(t_{0}\right)\right) \geq-\int_{\mathbb{R}} J(x) x \int_{0}^{1}\left[u(t-z x)-u\left(t_{0}-z x\right)\right] d z d x \\
\geq & -\int_{0}^{N} J(x) x \int_{0}^{1} u(t-z x) d z d x-\int_{-\infty}^{0} J(x)|x| \int_{0}^{1} u\left(t_{0}-z x\right) d z d x-x_{c} \int_{N}^{+\infty} J(x) x d x \\
\geq & -\int_{0}^{N} J(x) x \int_{0}^{1} u(t-z x) d z d x-u\left(t_{0}\right) \int_{-\infty}^{0} J(x)|x| d x-\varepsilon(N) \quad \text { for } t \geq t_{0},
\end{aligned}
$$


where $N>0$ is arbitrary and $\varepsilon$ satisfies (2.14). Passing to the limit as $t \rightarrow+\infty$, from the latter inequality it follows that

$$
u\left(t_{0}\right)\left(c-\int_{-\infty}^{0} J(x)|x| d x\right) \leq \varepsilon(N) \quad \text { for } N>0 .
$$

Consequently, in view of (2.14), the inequality (2.17) yields

$$
u\left(t_{0}\right)\left(c-\int_{-\infty}^{0} J(x)|x| d x\right) \leq 0 .
$$

Thus, on account of $u\left(t_{0}\right)>0$ we have

$$
c \leq \int_{-\infty}^{0} J(x)|x| d x
$$

which contradicts (2.11). The obtained contradiction proves the assertion of the lemma.

Proof of Theorem 1. According to Lemma 9, for every $u_{0} \in\left(0, e^{-\lambda h}\right)$ there exists a positive solution $u$ to (1.1) such that (2.10) holds (recall that $x_{c}=1+f_{0}\left(e^{\frac{h}{c}}-1\right)$ ). In view of Lemmas 10 and 12 we have that

$$
\text { either } \quad \lim _{t \rightarrow-\infty} u(t)=0 \quad \text { or } \quad \lim _{t \rightarrow+\infty} u(t)=0 .
$$

However, Lemma 13 assures that (2.16) is valid. Therefore, the theorem is proven.

\section{APPLiCATIONS AND EXAMPLES}

In this section, we present some examples to illustrate the application of the main results of this paper about the existence of traveling wave to equation (1.1) with some specific growth term $f$ and dispersal kernel $J$, which are usually used representing the dynamical population model of single species in ecology (see, e.g. $[3,5,6,13,18]$ ).

We consider the exponential kernel

$$
J_{\alpha}(s)=\frac{e^{-\frac{(s+\rho)^{2}}{4 \alpha}}}{\sqrt{4 \pi \alpha}}, \quad \rho \geq 0 .
$$

Note that $\int_{\mathbb{R}} J_{\alpha}(x) d x=1$ and $\int_{-\infty}^{0} J_{\alpha}(x) e^{-\lambda x} d x<+\infty$ for all $\lambda>0$. In addition, by computation we have

$$
\int_{-\infty}^{+\infty} J_{\alpha}(x)|x| d x=\sqrt{\frac{4 \alpha}{\pi}} e^{-\frac{\rho^{2}}{4 \alpha}}+\rho \operatorname{erf}\left(\frac{\rho}{\sqrt{4 \alpha}}\right)=: M_{1}<+\infty,
$$

where $\operatorname{erf}(x)$ is the Gauss error function. Moreover, we also get

$$
\int_{0}^{+\infty} J_{\alpha}(x) d x=\frac{1}{2}\left(1-\operatorname{erf}\left(\frac{\rho}{\sqrt{4 \alpha}}\right)\right)=: M_{2},
$$




$$
\int_{-\infty}^{0} J_{\alpha}(x) e^{-\lambda x} d x=\frac{e^{\lambda(\rho+\lambda \alpha)}}{2}\left(1+\operatorname{erf}\left(\frac{\rho+2 \lambda \alpha}{\sqrt{4 \alpha}}\right)\right)=: M_{3}(\lambda) \text { for } \lambda>0 .
$$

Let $f$ be as in Section 1, and let $f_{0}, x_{c}$, and $f_{c}$ be as in Section 2. Put

$$
H(\lambda):=\int_{0}^{+\infty} J_{\alpha}(x) d x+\int_{-\infty}^{0} J_{\alpha}(x) e^{-\lambda x} d x, \quad G_{c}(\lambda):=c \lambda-f_{c}, \quad \lambda \geq 0 .
$$

Note that $H(0)=1, H^{\prime \prime}(\lambda)>0$ for $\lambda>0, H^{\prime}(0)=-\int_{-\infty}^{0} x J_{\alpha}(x) d x>0$ and $H(\lambda)$ does not depend on $c$. Moreover, $G_{c}(0)=-f_{c}<0, G_{c}^{\prime}(\lambda)=c>0$ and $G_{c}$ is increasing with respect to $c$ at every positive point $\lambda$. In consequence, there exist $c_{\star}, \lambda_{\star}>0$ such that

$$
G_{c_{\star}}\left(\lambda_{\star}\right)=H\left(\lambda_{\star}\right), \quad G_{c_{\star}}(\lambda)<H(\lambda) \text { for } \lambda>0, \lambda \neq \lambda_{\star} .
$$

In addition, for each $c>c_{\star}$, we have

$$
G_{c}\left(\lambda_{\star}\right)>H\left(\lambda_{\star}\right)
$$

and, if $c<c_{\star}$, then $G_{c}\left(\lambda_{\star}\right)<H\left(\lambda_{\star}\right)$. By substitution, we finally have, for each speed $c \geq c_{\star}$,

$$
c \lambda_{\star} \geq M_{2}+M_{3}\left(\lambda_{\star}\right)+f_{c} .
$$

We also obatin that $G_{c_{\star}}^{\prime}\left(\lambda_{\star}\right)=H^{\prime}\left(\lambda_{\star}\right)$, i.e.,

$$
c_{\star}=-\int_{-\infty}^{0} x J_{\alpha}(x) e^{-\lambda_{\star} x} d x
$$

Now, to estimate the right-hand term of (3.1), we will consider a particular nonlinearity. We study two types of nonlinearities, usually used in literature for nondegenerate case $\left(f^{\prime}(0)>0\right)$ and degenerate case $\left(f^{\prime}(0)=0\right)$.

Example 3.1 Let $f$ be the classical Fisher-KPP nonlinearity given by $f(s)=s(1-$ $s)$ for $s \geq 0$. Thus $f_{0}=\frac{1}{4}, x_{c}=\frac{1}{4}\left(3+e^{\frac{h}{c}}\right)>1$, and $f_{c}=\left|f^{\prime}\left(x_{c}\right)\right|=\frac{1+e^{h / c}}{2}$. According to the above-proven, there exist positive numbers $c_{\star}$ and $\lambda_{\star}$ such that

$$
c_{\star} \lambda_{\star}=M_{2}+M_{3}\left(\lambda_{\star}\right)+f_{c_{\star}}
$$

and (3.1) holds for every $c \geq c_{\star}$. Substituting into (3.1) we obtain

$$
c \lambda_{\star} \geq M_{2}+M_{3}\left(\lambda_{\star}\right)+\frac{1+e^{h / c}}{2} \quad \text { for } c \geq c_{\star} .
$$

Moreover, the function

$$
P_{c}(\lambda):=1+f_{c} e^{\lambda h}=1+\frac{1+e^{h / c}}{2} e^{\lambda h}
$$

is increasing with respect to $\lambda$, and decreasing with respect to $c$ at every positive point $\lambda$. Therefore, there exits $c_{*} \geq c_{\star}$ such that

$$
c_{*} \lambda_{\star}=\max \left\{M_{2}+M_{3}\left(\lambda_{\star}\right)+\frac{1+e^{h / c_{*}}}{2}, P_{c_{*}}\left(\lambda_{\star}\right)\right\} .
$$


Consequently,

$$
c \lambda_{\star} \geq \max \left\{M_{2}+M_{3}\left(\lambda_{\star}\right)+\frac{1+e^{h / c}}{2}, P_{c}\left(\lambda_{\star}\right)\right\} \quad \text { for } c \geq c_{*} .
$$

Set

$$
M=\frac{1}{\lambda_{\star}} \max \left\{M_{2}+M_{3}\left(\lambda_{\star}\right)+\frac{1+e^{h / c_{*}}}{2}, P_{c_{*}}\left(\lambda_{\star}\right), \lambda_{\star} M_{1}\right\} .
$$

Then the proof of Theorem 1 guarantees the following result.

Theorem 2. For every $c>M$ there exists a positive solution $u$ to the monostable delay equation

$$
c u^{\prime}(t)=J_{\alpha} \star u(t)-u(t)+u(t-h)(1-u(t-h)),
$$

satisfying

$$
\lim _{t \rightarrow-\infty} u(t)=0, \quad e^{-\lambda_{\star} h} \leq \liminf _{t \rightarrow+\infty} u(t) \leq \sup _{t \in \mathbb{R}} u(t) \leq 1+\frac{e^{\frac{h}{c}}-1}{4} .
$$

Example 3.2 We consider the nonlinearity $f(s)=s^{p+1}(1-s), p>0$ (degenerate case). Then

$$
f_{0}=\frac{(p+1)^{p+1}}{(p+2)^{p+2}}, \quad x_{c}=1+\frac{(p+1)^{p+1}\left(e^{\frac{h}{c}}-1\right)}{(p+2)^{p+2}}
$$

and

$$
\begin{aligned}
f_{c}= & \left|f^{\prime}\left(x_{c}\right)\right| \\
& =\left(1+\frac{(p+1)^{p+1}\left(e^{\frac{h}{c}}-1\right)}{(p+2)^{p+2}}\right)^{p}\left(1+\frac{(p+1)^{p+1}\left(e^{\frac{h}{c}}-1\right)}{(p+2)^{p+1}}\right)=: N_{1}(c) .
\end{aligned}
$$

According to the above-proven, there exist positive numbers $c_{\star}$ and $\lambda_{\star}$ such that (3.2) is fulfilled and (3.1) holds for every $c \geq c_{\star}$. Substituting into (3.1) we obtain

$$
c \lambda_{\star} \geq M_{2}+M_{3}\left(\lambda_{\star}\right)+N_{1}(c) \quad \text { for } c \geq c_{\star} .
$$

Moreover, the function

$$
\bar{P}_{c}(\lambda):=1+f_{c} e^{\lambda h}=1+N_{1}(c) e^{\lambda h}
$$

is increasing with respect to $\lambda$ and decreasing with respect to $c$ at every positive point $\lambda$. Therefore, there exits $c_{*} \geq c_{\star}$ such that

$$
c_{*} \lambda_{\star}=\max \left\{M_{2}+M_{3}\left(\lambda_{\star}\right)+N_{1}\left(c_{*}\right), \bar{P}_{c_{*}}\left(\lambda_{\star}\right)\right\} .
$$

Consequently,

$$
c \lambda_{\star} \geq \max \left\{M_{2}+M_{3}\left(\lambda_{\star}\right)+N_{1}(c), \bar{P}_{c}\left(\lambda_{\star}\right)\right\} \quad \text { for } c \geq c_{*} .
$$


Now, set

$$
N=\frac{1}{\lambda_{\star}} \max \left\{M_{2}+M_{3}\left(\lambda_{\star}\right)+N_{1}\left(c_{*}\right), \bar{P}_{c_{*}}\left(\lambda_{\star}\right), \lambda_{\star} M_{1}\right\} .
$$

Then we have the following result.

Theorem 3. For every $c>N$ there exists a positive solution $u$ to the monostable delay equation

$$
c u^{\prime}(t)=J_{\alpha} \star u(t)-u(t)+(u(t-h))^{p+1}(1-u(t-h)),
$$

satisfying

$$
\lim _{t \rightarrow-\infty} u(t)=0, \quad e^{-\lambda \star h} \leq \liminf _{t \rightarrow+\infty} u(t) \leq \sup _{t \in \mathbb{R}} u(t) \leq 1+\frac{(p+1)^{p+1}\left(e^{\frac{h}{c}}-1\right)}{(p+2)^{p+2}} .
$$

\section{REFERENCES}

[1] M. Aguerrea, C. Gomez, and S. Trofimchuk, "On uniqueness of semi-wavefronts," Math. Ann., vol. 354, no. 1, pp. 73-109, 2012, doi: 10.1007/s00208-011-0722-8. [Online]. Available: https://doi.org/10.1007/s00208-011-0722-8

[2] M. Alfaro, "Fujita blow up phenomena and hair trigger effect: the role of dispersal tails," Ann. Inst. H. Poincaré Anal. Non Linéaire, vol. 34, no. 5, pp. 1309-1327, 2017, doi: 10.1016/j.anihpc.2016.10.005. [Online]. Available: https://doi.org/10.1016/j.anihpc.2016.10.005

[3] M. Alfaro, "Slowing Allee effect versus accelerating heavy tails in monostable reaction diffusion equations," Nonlinearity, vol. 30, no. 2, pp. 687-702, 2017, doi: 10.1088/1361-6544/aa53b9. [Online]. Available: https://doi.org/10.1088/1361-6544/aa53b9

[4] M. Alfaro and J. Coville, "Propagation phenomena in monostable integro-differential equations: acceleration or not?" J. Differential Equations, vol. 263, no. 9, pp. 5727-5758, 2017, doi: 10.1016/j.jde.2017.06.035. [Online]. Available: https://doi.org/10.1016/j.jde.2017.06.035

[5] O. Bonnefon, J. Coville, J. Garnier, F. Hamel, and L. Roques, "The spatio-temporal dynamics of neutral genetic diversity,” Ecological Complexity, vol. 20, pp. 282-292, 2014. [Online]. Available: https://hal.archives-ouvertes.fr/hal-01257407

[6] J. Carr and A. Chmaj, "Uniqueness of travelling waves for nonlocal monostable equations," Proc. Amer. Math. Soc., vol. 132, no. 8, pp. 2433-2439, 2004, doi: 10.1090/S0002-9939-04-07432-5. [Online]. Available: https://doi.org/10.1090/S0002-9939-04-07432-5

[7] J. Coville, J. Dávila, and S. Martínez, "Nonlocal anisotropic dispersal with monostable nonlinearity," J. Differential Equations, vol. 244, no. 12, pp. 3080-3118, 2008, doi: 10.1016/j.jde.2007.11.002. [Online]. Available: https://doi.org/10.1016/j.jde.2007.11.002

[8] J. Coville and L. Dupaigne, "On a non-local equation arising in population dynamics," Proc. Roy. Soc. Edinburgh Sect. A, vol. 137, no. 4, pp. 727-755, 2007, doi: 10.1017/S0308210504000721. [Online]. Available: https://doi.org/10.1017/S0308210504000721

[9] J. Garnier, "Accelerating solutions in integro-differential equations," SIAM J. Math. Anal., vol. 43, no. 4, pp. 1955-1974, 2011, doi: 10.1137/10080693X. [Online]. Available: https://doi.org/10.1137/10080693X

[10] R. Hakl, A. Lomtatidze, and B. Půža, "On nonnegative solutions of first order scalar functional differential equations," Mem. Differential Equations Math. Phys., vol. 23, pp. 51-84, 2001.

[11] R. Hakl, A. Lomtatidze, and B. Půža, "On a boundary value problem for first-order scalar functional differential equations," Nonlinear Anal., vol. 53, no. 3-4, pp. 391-405, 2003, doi: 
10.1016/S0362-546X(02)00305-X. [Online]. Available: https://doi.org/10.1016/S0362-546X(02) 00305-X

[12] R. Huang, M. Mei, and Y. Wang, "Planar traveling waves for nonlocal dispersion equation with monostable nonlinearity," Discrete Contin. Dyn. Syst., vol. 32, no. 10, pp. 3621-3649, 2012, doi: 10.3934/dcds.2012.32.3621. [Online]. Available: https://doi.org/10.3934/dcds.2012.32.3621

[13] V. Ortega-Cejas, J. Fort, and V. Méndez, "The role of the delay time in the modeling of biological range expansions," Ecology, vol. 85, no. 1, pp. 258-264, 2004, doi: 10.1890/02-0606. [Online]. Available: https://esajournals.onlinelibrary.wiley.com/doi/abs/10.1890/02-0606

[14] S. Pan, W.-T. Li, and G. Lin, "Existence and stability of traveling wavefronts in a nonlocal diffusion equation with delay," Nonlinear Anal., vol. 72, no. 6, pp. 3150-3158, 2010, doi: 10.1016/j.na.2009.12.008. [Online]. Available: https://doi.org/10.1016/j.na.2009.12.008

[15] Z. Vorel, "Continuous dependence on parameters," Nonlinear Anal., vol. 5, no. 4, pp. 373-380, 1981, doi: 10.1016/0362-546X(81)90021-3. [Online]. Available: https: //doi.org/10.1016/0362-546X(81)90021-3

[16] H. F. Weinberger, "Long-time behavior of a class of biological models," SIAM J. Math. Anal., vol. 13, no. 3, pp. 353-396, 1982, doi: 10.1137/0513028. [Online]. Available: https://doi.org/10.1137/0513028

[17] G.-B. Zhang, "Traveling waves in a nonlocal dispersal population model with age-structure," Nonlinear Anal., vol. 74, no. 15, pp. 5030-5047, 2011, doi: 10.1016/j.na.2011.04.069. [Online]. Available: https://doi.org/10.1016/j.na.2011.04.069

[18] G.-B. Zhang, W.-T. Li, and Z.-C. Wang, "Spreading speeds and traveling waves for nonlocal dispersal equations with degenerate monostable nonlinearity," J. Differential Equations, vol. 252, no. 9, pp. 5096-5124, 2012, doi: 10.1016/j.jde.2012.01.014. [Online]. Available: https://doi.org/10.1016/j.jde.2012.01.014

[19] G.-B. Zhang and R. Ma, "Spreading speeds and traveling waves for a nonlocal dispersal equation with convolution-type crossing-monostable nonlinearity," Z. Angew. Math. Phys., vol. 65, no. 5, pp. 819-844, 2014, doi: 10.1007/s00033-013-0353-x. [Online]. Available: https://doi.org/10.1007/s00033-013-0353-x

Authors' addresses

Maitere Aguerrea

Facultad de Ciencias Básicas, Universidad Católica del Maule, Casilla 617, Talca, Chile

E-mail address: maguerrea@ucm.cl

\section{Robert Hakl}

Institute of Mathematics, Czech Academy of Sciences, Žižkova 22, 61662 Brno, Czech Republic

E-mail address: hakl@ipm.cz 